\title{
Comparación de diferentes agentes plastificantes adicionados en esponjas de quitina obtenidas de cefalotórax de camarón
}

OSCAR, ARIAS-TORRES ', ANA M., MENDOZA-MÁRQUEZ', EVELYN M., BÁRCENAS-OCHOA', RONNY,

FLORES², ROLANDO S., GARCÍA-GÓMEZI', MARÍA DEL CARMEN, DURÁN-DOMÍNGUEZ-DE-BAZÚA I*

' Laboratorios E301, E302 y E303 de Ingeniería Química Ambiental y de Química Ambiental (LLAyQA), Conjunto "E",

Facultad de Química, UNAM (Universidad Nacional Autónoma de México). México, D.F. México.

${ }^{2}$ Laboratorio de Química Ambiental, Facultad de Ciencias Químicas, Universidad Central del Ecuador, Quito.

*Correspondencia: mcduran@unam.mx

Recibido: 20 enero 2012 / Aceptado: 03 octubre 2012

\section{Resumen}

En esta investigación se utilizaron residuos de camarón, cefalotórax y exoesqueletos, para extraer quitina y obtener esponjas usando un proceso de química verde (․ Se probó la inclusión de tres agentes plastificantes: polietilenglicol acuoso al 10\%, sorbitol acuoso al $70 \%$ y propilenglicol acuoso en una concentración desconocida (muestra comercial) para verificar la resistencia del biopolímero. Se estudiaron dos grupos (con/sin la adición de agentes plastificantes) empleando las metodologías establecidas por la ASTM: tensión en la carga máxima, tensión en el punto de ruptura, módulo de Young, porcentaje de deformación, límite elástico y tenacidad. Se calculó el límite elástico desde los datos experimentales. El control fue una probeta de poliestireno espumado. El análisis de los resultados entre grupos $(p<0.05)$ indicó que la inclusión de los agentes plastificantes favorecieron las características mecánicas de las esponjas con respecto de la probeta de poliestireno usada como referencia, siendo el sorbitol el agente que no presentó diferencias significativas con el control en las pruebas de tensión en la carga máxima, tensión de ruptura, módulo de Young, \% de deformación y límite elástico ( $p<0.05)$. Dado que sí hubo diferencias significativas $(p<0.05)$ con respecto de la tenacidad, se continuará la investigación para buscar nuevos y mejores plastificantes.

Palabras clave: Cefalotórax-exoesqueleto de camarón, quitina, esponja de quitina, agentes plastificantes, química verde.

\section{Comparison between chitin sponges cephalothorax shrimp covered with different plasticizers}

\section{Abstract}

In this study, shrimp exoskeletons and cephalothorax were used for the extraction of chitin and the production of sponges using a green chemistry process $(0$. The inclusion of three plasticizing agents, $10 \%$ aqueous polyethylene glycol, 70\% aqueous sorbitol, and propylene glycol at an unknown concentration (commercial sample), was tested to evaluate the biopolymer resistance. Two test groups (with/without plasticizers addition) were analyzed using the ASTM Testing: Energy of rupture tension, rupture tension, Young's modulus, \% rupture elongation, and toughness. From experimental data tensile strength was calculated. The control was a cylinder cast with polystyrene foam. The analysis of results between groups $(p<0.05)$ showed that the inclusion of plasticizers favored the mechanical characteristics of the sponges with respect to the sample of polystyrene (control), except for the sorbitol agent, which had no significant differences with the control $(p<0.05)$ in energy of rupture tension, rup- 
ture tension, Young's modulus, \% rupture elongation, and tensile strength. As there were significant differences $(p<0.05)$ with respect to toughness, research is being carried out to find new and better plastifying agents.

Key words: Shrimp cephalothorax-exoskeletons, chitin, chitin sponge, plastifying agents, green chemistry.

\section{Introducción}

México es considerado uno de los diez países de mayor producción de camarón a nivel mundial [1]. De un camarón entero, sólo es aprovechado alrededor del $50 \%$ ya que el resto es considerado material de desperdicio (cefalotórax y exoesqueleto). Uno de los principales componentes de este animal, es un polímero biológico natural: la quitina, que es el segundo polímero más abundante en la naturaleza, después de la celulosa [2]. En este estudio se planteó la posibilidad de crear un polímero espumado elaborado a partir de quitina, como una alternativa de los polímeros sintéticos, utilizando tecnologías más limpias y que no dañen el ambiente como las tradicionales en donde se utilizan $\mathrm{HCl}$ y $\mathrm{NaOH}$ [3] . Estas esponjas podrían sustituir a los polímeros sintéticos como el poliuretano y el poliestireno para la elaboración de empaques biodegradables. Para ello se propuso la adición de tres diferentes agentes aditivos como se muestran en la Figura 1 y que no modifican el periodo de biodegradabilidad de los polímeros elaborados a base de quitina que, según investigaciones previas, es de dos semanas al contacto con la basura, bajo las condiciones normales de un sitio de disposición controlada de desechos urbanos [4]. En algunos casos, como el sorbitol, los aditivos pueden acelerar este proceso de degradación [5] [6].<smiles>C[CH]OCCC(C)O</smiles>

Polietilenglicol

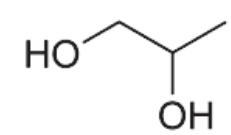

Propilenglicol<smiles>OC[C@H](O)[C@@H](O)[C@H](O)[C@H](O)CO</smiles>

Figura 1. Estructuras químicas de los agentes aditivos utilizados

\section{Materiales y métodos}

\subsection{Obtención y limpieza de la materia prima}

Se emplearon cefalotórax y exoesqueletos de camarón fresco, proporcionados gratuitamente por los comerciantes de la zona de pescados y mariscos de "La Nueva Viga", en la ciudad de México, D.F. Una vez adquiridos, se lavaron con un chorro de agua de la llave, hasta observar que el agua saliera limpia, dejándose escurrir durante 20 minutos.

\subsection{Separación y formación de las esponjas de quitina}

La separación de quitina de los subproductos de camarón y su disolución, se realizó siguiendo la metodología propuesta por Flores [7] [8], donde, una vez obtenidos los exoesqueletos del cefalotórax de camarón $(C C)$, se limpian y se lavan bajo un chorro de agua de la llave. Posteriormente, se mezclan en una licuadora a $1.000 \mathrm{rpm}$, adicionándose los exoesqueletos limpios y el agua en una relación 1:2 en masa. La pasta obtenida se filtra con un tamiz de tamaño de malla de $1 \mathrm{~mm}$ de abertura. Los sólidos retenidos en el tamiz se secan en una estufa eléctrica a $60^{\circ} \mathrm{C}$ durante 18 horas. La harina obtenida de exoesqueletos de camarón parcialmente desproteinizado (EPD), se tamiza con la finalidad de obtener un tamaño de partícula homogéneo de $149 \mu \mathrm{m}<\mathrm{P}<2 \mathrm{~mm}$ y se conserva en refrigeración dentro de un envase hermético. Posteriormente, se prepara el disolvente MAC (mezcla de Metanol-Agua-Cloruro de Calcio 1:4:1) para disolver la quitina presente en el EPD. La quitina presente en los exoesqueletos es disuelta en esta mezcla MAC-1:4:1, para permitir su extracción. La mezcla de harina de EPD con MAC se coloca en frascos de $100 \mathrm{~mL}$, con tapa hermética, se homogeneíza y se deja en reposo a $25^{\circ} \mathrm{C}$ en una incubadora durante 48 horas con el objetivo de que se forme el complejo soluble quitina-calcio. Transcurrido este tiempo, el sobrenadante (QUIMAC o quitina en solución), se filtra 
al vacío en equipo millipore, utilizando una membrana polimérica con la finalidad de separar los residuos sólidos de EPD. Las soluciones QUIMAC se pesan y emplean para la obtención de las esponjas de quiti$\mathrm{na}$, en donde se agitan manualmente durante tres minutos, con una varilla de vidrio, hasta la producción de espuma. La espuma QUIMAC se coloca en moldes rectangulares de $22 \mathrm{~cm}$ de largo $\times 15 \mathrm{~cm}$ de ancho y se guardan en una cámara de humidificación con una medida relativa mayor al $90 \%$ para que el cloruro de calcio, absorba agua del ambiente y la quitina precipite en forma de esponja. Transcurridas 48 horas, el disolvente separado es extraído, dejando en el molde a la esponja de quitina (SPQ), la cual se lava con agua destilada y se procede a secar a temperatura ambiente. Una vez obtenidas las esponjas de quitina, son pesadas y medidas.

\subsection{Inclusión del agente aditivo}

Para definir el mejor agente plastificante que sirviera para proporcionar las mejores propiedades mecánicas de las esponjas de quitina, se diseñó un experimento con tres diferentes niveles de concentración de cada uno de ellos, considerando como variable dependiente las propiedades mecánicas de las esponjas obtenidas. De los tres agentes plastificantes, solamente el polietilenglicol, fue acondicionado (preparación del agente plastificante con agua, con una relación de $100 \mathrm{~g}$ de plastificante en $1000 \mathrm{~mL}$ de agua, según el instructivo del fabricante), ya que los otros dos plastificantes: sorbitol y propilenglicol, sí venían en solución. En el caso del sorbitol, se encontraba en un porcentaje del $70 \%$ en disolución acuosa. El propilenglicol fue adquirido en la farmacia "París", del centro de la Ciudad de México, sin ninguna especificación por parte del proveedor de su contenido en agua. Esta incógnita no afectó su aplicación en estos experimentos. Debido a que la masa promedio de las probetas de esponja de quitina era de $0.1 \mathrm{~g}$, la adición del agente plastificante se realizó de manera individual sobre las probetas ya cortadas, dejándolas en contacto con la solución por un lapso de 24 horas. En la Tabla 1, se presentan las proporciones en $\mathrm{g}$ de esponja/mL de plastificante: Nivel bajo 1:1; Nivel medio 1:10; Nivel alto $1: 100$, con un lote sin plastificante.
Tabla 1. Proporción de los agentes plastificantes con relación a la masa de las probetas de esponja de quitina

\begin{tabular}{cccc}
\hline $\begin{array}{c}\text { Agente } \\
\text { plastificante }\end{array}$ & $\begin{array}{c}\text { Polietilenglicol } \\
(\mathbf{m L} / \mathbf{g ~ E Q})\end{array}$ & $\begin{array}{c}\text { Sorbitol } \\
\text { acuoso al 70\% } \\
(\mathbf{m L} / \mathbf{g ~ E Q})\end{array}$ & $\begin{array}{c}\text { Propilenglicol } \\
(\mathbf{m L} / \mathbf{g ~ E Q})\end{array}$ \\
\hline $\begin{array}{c}\text { Sin agente } \\
\text { plastificante }\end{array}$ & 0 & 0 & 0 \\
Nivel bajo & 1 & 1 & 1 \\
Nivel medio & 10 & 10 & 10 \\
Nivel alto & 100 & 100 & 100 \\
\hline
\end{tabular}

${ }^{*} \mathrm{EQ}$ : Esponja de quitina

\subsection{Pruebas mecánicas}

Una vez obtenidas las esponjas que presentaron una mayor dureza (determinada de manera subjetiva: visual y táctil), se prepararon probetas de quitina y poliestireno de $40 \mathrm{~mm} \times 10 \mathrm{~mm}$. Dichas probetas se cortaron con un "suaje" o molde y se tomaron medidas de la anchura y el espesor con un Vernier (Figura 2). Cada probeta se probó en un equipo Instron Modelo 5500R, en el Instituto de Investigaciones en Materiales de la UNAM, a una velocidad de cruceta de $1 \mathrm{~mm} /$ min y una distancia inicial de $30 \mathrm{~mm}$, bajo condiciones ambientales de $50 \%$ de humedad y de 22.7 ${ }^{\circ} \mathrm{C}\left(73^{\circ} \mathrm{F}\right)$. Con este equipo, fue posible la obtención de los siguientes datos: tensión en la carga máxima $(\mathrm{MPa})$, tensión en el punto de ruptura (MPa), módulo de Young (MPa), porcentaje de deformación (\%) y la tenacidad. De los datos experimentales, obtenidos se pudo calcular una sexta propiedad mecánica: el límite elástico [9].

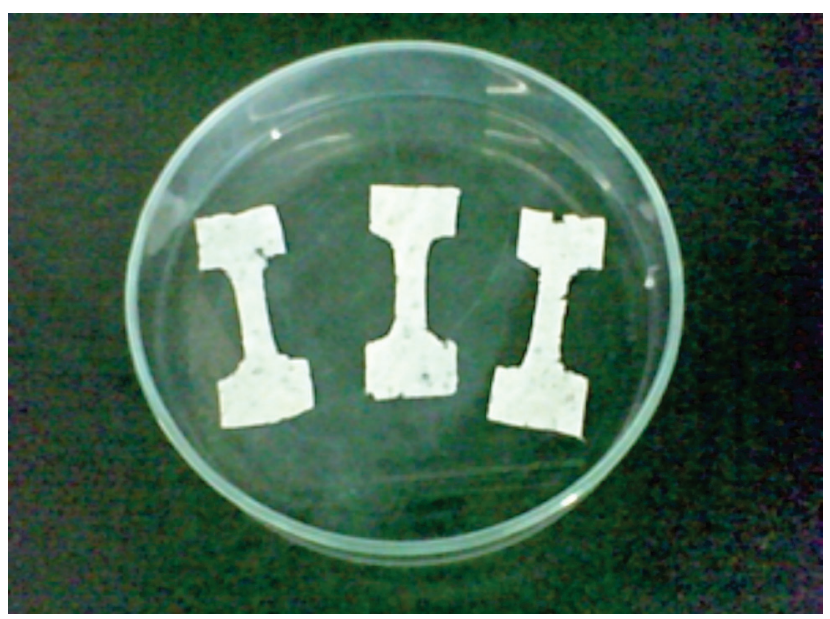

Figura 2. Probetas de esponja de quitina acondicionadas con aditivos 
2.5 Comparación entre una probeta de esponja de quitina $y$ una de poliestireno espumado

Después de realizar las pruebas mecánicas para ambos grupos, los resultados fueron sometidos a un análisis de varianza utilizando el software Statgraphics Plus versión 5.1. Con esto se pudieron analizar y comparar los diferentes valores obtenidos en las cinco pruebas mecánicas realizadas para cada una de las probetas acondicionadas con los tres aditivos, así como los valores de las pruebas mecánicas para las probetas de poliestireno espumado de uso comercial que se emplearon como control.

\section{Resultados y discusión}

\subsection{Porcentaje de rendimiento de harina obtenida a partir de exoesqueletos frescos}

Para la elaboración de la harina de exoesqueleto-cefalotórax parcialmente desproteinizado, EPD, se obtuvieron los subproductos de camarón del nuevo mercado de "La Viga", en la Ciudad de México, realizándose dos ensayos para conseguir la harina (cada ensayo con una muestra de harina) con la finalidad de evaluar la eficiencia de separación (cefalotórax transformado en harina seca). Se pesaron los kilogramos para cada ensayo como se aprecia en la Tabla 2. En ella se presenta la producción de harina de $1 \mathrm{~mm}$ de tamaño promedio de partícula de exoesqueleto de camarón a partir de residuos frescos, que traen todavía órganos internos. El porcentaje de rendimiento está calculado a partir de exoesqueletos-cefalotórax frescos de camarón.

\subsection{Elaboración de esponjas de quitina utilizando los tres agentes aditivos}

Los cefalotórax del lote 1 (Tabla 2) fueron los que arrojaron un mayor porcentaje de rendimiento al momento de realizar el proceso de limpieza, siendo el lote utilizado para preparar las probetas en la realización de las pruebas mecánicas las cuales se aprecian en la Tabla 3.

Tabla 2. Obtención de harina de cefalotórax y exoesqueleto

\begin{tabular}{ccccc}
\hline $\begin{array}{c}\text { No. de } \\
\text { Lote/Muestra, } \boldsymbol{g}\end{array}$ & $\begin{array}{c}\text { Cefalotórax y exoesqueleto limpio } \\
\text { húmedo sin órganos internos, } \boldsymbol{g}\end{array}$ & $\begin{array}{c}\% \text { de } \\
\text { rendimiento }\end{array}$ & $\begin{array}{c}\text { Cefalotórax y exoesqueleto } \\
\text { seco molido (harina), g }\end{array}$ & $\begin{array}{c}\% \text { de } \\
\text { rendimiento }\end{array}$ \\
\hline $1 / 7,000$ & 524 & 7 & 182.1 & 34.75 \\
$2 / 30,000$ & 1420 & 4.7 & 519.5 & 36.58 \\
\hline
\end{tabular}

Tabla 3. Obtención de esponjas de quitina para las pruebas mecánicas

\begin{tabular}{|c|c|c|c|c|c|c|c|}
\hline $\begin{array}{c}\text { Adición de } \\
\text { plastificante }\end{array}$ & $\begin{array}{l}\text { Masa } \\
\text { CPD } \\
(g)\end{array}$ & $\begin{array}{c}\text { Masa } \\
\text { MAC-141 } \\
\text { (g) }\end{array}$ & $\begin{array}{c}\text { Masa } \\
\text { QUIMAC* } \\
\text { (g) }\end{array}$ & $\begin{array}{c}\text { Masa } \\
\text { esponja } \\
(g)\end{array}$ & $\begin{array}{c}\text { Volumen } \\
\text { de aditivo } \\
(\mathrm{mL})\end{array}$ & $\begin{array}{l}\text { Diámetro del tamaño } \\
\text { de la esponja } \\
(\mathrm{mm})\end{array}$ & $\begin{array}{c}\text { Rendimiento partiendo } \\
\text { de g iniciales de CPD } \\
(\%)\end{array}$ \\
\hline Propilenglicol & 12.03 & 240.34 & 125.45 & 7.31 & 73 & 45.90 & 67.76 \\
\hline Sorbitol & 12.07 & 241.63 & 113.97 & 6.41 & 64 & 48.02 & 53.10 \\
\hline Polietilenglicol & 12.00 & 240.07 & 114.31 & 6.42 & 62 & 49.24 & 53.50 \\
\hline
\end{tabular}

3.3 Resultados de las pruebas mecánicas a las probetas de quitina y poliestireno espumado (control)

Para la realización de las pruebas mecánicas, se fabricaron 15 esponjas de quitina con cada aditivo, de las cuales se tomaron tres probetas para cada una de las cinco pruebas mecánicas, teniendo así un total de 45 probetas con plastificante y 15 más sin plastificante, todas fabricadas a partir de exoesqueletos-cefalotórax de camarón. Las cinco pruebas realizadas en las espumas de quitina son las mencionadas en el método de la American Society for Testing and Materials [9]. En la Tabla 4 se muestra una comparación de las propiedades mecánicas de las probetas acondicionadas, del poliestireno puro y espumado y de las probetas de quitina sin la adición de agente plastificante. Utilizando los resultados promedio de cada una de las pro- 
piedades mecánicas realizadas, de acuerdo con los valores que se enlistan en esta tabla, se observó que los tres aditivos que se utilizaron, modificaron algunas de las propiedades mecánicas de las esponjas que fueron acondicionadas, siendo el sorbitol el que produjo espumas más semejantes a las probetas de poliestireno espumado usado como control. En la Tabla 5 se resu- men los resultados estadísticos obtenidos al evaluar las propiedades mecánicas de las probetas acondicionadas con los tres diferentes aditivos estudiados (propilenglicol, polietilenglicol y sorbitol, adicionados en una proporción $1: 10$ ) y elaboradas con quitina de origen natural y su comparación con un polímero sintético, el poliestireno espumado de uso comercial.

Tabla 4. Comparación de las propiedades mecánicas de las probetas acondicionadas con polietilenglicol, propilenglicol y sorbitol con probetas de poliestireno puro y espumado y con las probetas de espuma de quitina sin acondicionar

\begin{tabular}{cccccc}
\hline \multicolumn{7}{c}{ Comparación de propiedades mecánicas } \\
\hline Material & $\begin{array}{c}\text { Tensión de } \\
\text { ruptura (MPa) }\end{array}$ & $\begin{array}{c}\% \text { De } \\
\text { deformación }\end{array}$ & $\begin{array}{c}\text { Módulo de } \\
\text { Young }(\mathrm{MPa})\end{array}$ & $\begin{array}{c}\text { Límite } \\
\text { elástico }(\mathrm{MPa})\end{array}$ & $\begin{array}{c}\text { Tenacidad } \\
(\mathrm{MPa})\end{array}$ \\
\hline SPQ & 0.11 & 33.93 & 3.77 & 0.2 & 0.040 \\
SPQ-PG & 0.37 & 6.55 & 14.63 & 6.33 & 0.009 \\
SPQ-S & 1.28 & 3.42 & 50.31 & 3.90 & 0.019 \\
SPQ-PEG & 0.67 & 3.39 & 49.67 & 2.68 & 0.005 \\
PS PURO & 48.71 & 6.08 & 1542 & & 1.610 \\
PS ESPUMADO & 1.14 & 6.60 & 35.42 & 6.88 & 0.040 \\
\hline PS: Poliestirno
\end{tabular}

\section{PS: Poliestireno}

$S P Q:$ Esponja de quitina sin la adición de agente plastificante

SPQ-PG: Esponja de quitina acondicionada con propilenglicol

SPQ-S: Esponja de quitina acondicionada con sorbitol

SPQ-PEG: Esponja de quitina acondicionada con polietilenglicol

Tabla 5. Resumen del análisis de varianza para los diferentes aditivos

\begin{tabular}{|c|c|c|c|c|}
\hline Control & $\begin{array}{c}\text { Agente } \\
\text { plastificante } \\
\text { (aditivo) }\end{array}$ & $\begin{array}{l}\text { Propiedad } \\
\text { mecánica }\end{array}$ & $\begin{array}{c}\text { Existe diferencia } \\
\text { estadísticamente } \\
\text { significativa }(a \geq 0.05)\end{array}$ & $\begin{array}{l}\text { No existe diferencia } \\
\text { estadísticamente } \\
\text { significativa ( } a \geq 0.05)\end{array}$ \\
\hline Poliestireno espumado & sorbitol & Tensión en la carga máxima & & $\mathrm{X}$ \\
\hline poliestireno espumado & sorbitol & Tensión en el punto de ruptura & & $\mathrm{x}$ \\
\hline poliestireno espumado & sorbitol & Módulo de Young & & $x$ \\
\hline poliestireno espumado & sorbitol & Porcentaje de deformación & & $\mathrm{x}$ \\
\hline poliestireno espumado & sorbitol & Tenacidad & $x$ & \\
\hline poliestireno espumado & sorbitol & Límite elástico & & $\mathrm{X}$ \\
\hline Poliestireno espumado & propilenglicol & Tensión en la carga máxima & $x$ & \\
\hline poliestireno espumado & propilenglicol & Tensión en el punto de ruptura & $x$ & \\
\hline poliestireno espumado & propilenglicol & Módulo de Young & & $\mathrm{X}$ \\
\hline poliestireno espumado & propilenglicol & Porcentaje de deformación & & $\mathrm{x}$ \\
\hline poliestireno espumado & propilenglicol & Tenacidad & $\mathrm{x}$ & \\
\hline poliestireno espumado & propilenglicol & Límite elástico & & $\mathrm{x}$ \\
\hline poliestireno espumado & polietilenglicol & Tensión en la carga máxima & $x$ & \\
\hline poliestireno espumado & polietilenglicol & Tensión en el punto de ruptura & & $\mathrm{x}$ \\
\hline poliestireno espumado & polietilenglicol & Módulo de Young & & $x$ \\
\hline poliestireno espumado & polietilenglicol & Porcentaje de deformación & & $\mathrm{x}$ \\
\hline poliestireno espumado & polietilenglicol & Tenacidad & $\mathrm{x}$ & \\
\hline poliestireno espumado & polietilenglicol & Límite elástico & $x$ & \\
\hline
\end{tabular}


De las Tablas 4 y 5 puede verse que en las probetas acondicionadas con sorbitol, comparadas con las de poliestireno espumado, en cinco de las seis evaluaciones mecánicas realizadas, no se encontraron diferencias estadísticamente significativas. Las propiedades en las que no se encontraron diferencias estadísticamente significativas fueron: tensión en la carga máxima, tensión en el punto de ruptura, módulo de Young, porcentaje de deformación y límite elástico. La única propiedad mecánica en la que se encontró diferencia estadísticamente significativa fue la que correspondió a la tenacidad; siendo, por tanto, interesante reevaluar en experimentos futuros a este aditivo para tratar de mejorar la tenacidad de las espumas acondicionadas con el sorbitol o buscar otro agente que permita que las esponjas sí se comporten como las de poliestireno espumado.

En las mismas Tablas 4 y 5 se enlistan también las propiedades mecánicas evaluadas de las probetas acondicionadas con propilenglicol comparadas con las probetas de poliestireno espumado. De acuerdo con los resultados estadísticos obtenidos, se encontraron diferencias significativas en tres de las seis propiedades evaluadas. Estas propiedades fueron: tensión en la carga máxima, tensión en el punto de ruptura y tenacidad. Las pruebas mecánicas en las que no se encontró una diferencia estadísticamente significativa fueron: la del módulo de Young, en el porcentaje de deformación y en el límite elástico. Este aditivo no resultó tan efectivo como el sorbitol.

Por último, se encuentran las propiedades mecánicas correspondientes a las probetas acondicionadas con polietilenglicol, comparadas con las propiedades del poliestireno espumado, en donde se ve que no existió una diferencia estadísticamente significativa en tres de las propiedades evaluadas correspondientes a: la tensión en el punto de ruptura, módulo de Young y porcentaje de deformación. Las propiedades mecánicas en las que se encontraron diferencias estadísticamente significativas fueron: tensión en la carga máxima, tenacidad y en el límite elástico. Al igual que el anterior, no resultó tan efectivo como el sorbitol.

Del estudio comparativo estadístico (software Statgraphics Plus versión 5.1), que utiliza el método de las menores diferencias significativas de Fisher, LSD, por sus siglas en inglés, para las medias de todas ellas, fue posible discernir cuál de los tres aditivos estudiados dio las probetas con las mejores características mecánicas cuando fueron comparadas con las del control de poliestireno espumado.

Para la tensión a la ruptura (Tabla 4), se encontró que el valor de esta propiedad, para las probetas acondicionadas con sorbitol, fue de 1.28MPa y para las probetas elaboradas con poliestireno espumado fue de 1.14MPa. Los valores obtenidos fueron muy semejantes, siendo mayor el valor obtenido por las probetas acondicionadas con sorbitol, por lo que dichas probetas, presentaron una mayor resistencia a romperse que las elaboradas con poliestireno espumado.

Para la propiedad de porcentaje de deformación, las probetas que obtuvieron un valor numérico semejante a las elaboradas con poliestireno espumado, fueron las acondicionadas con propilenglicol, con un valor de $6.552 \%$ mientras que las de poliestireno espumado, presentaron un valor de $6.60 \%$, por lo que ambas probetas tienen una resistencia a deformarse muy similar.

Para la propiedad del módulo de Young, los valores de las probetas acondicionadas no fueron semejantes a las de poliestireno espumado, dos de las probetas acondicionadas presentaron un valor mayor. Éste fue el caso de las acondicionadas con sorbitol (50.31 MPa) y las acondicionadas con polietilenglicol (49.67MPa), comparadas con el valor obtenido con las elaboradas con poliestireno espumado (35.42MPa). Al ser estos valores mayores, se concluye, que las probetas acondicionadas presentan una mayor rigidez que las elaboradas con poliestireno espumado.

Para la propiedad de límite elástico, las probetas acondicionadas con propilenglicol $(6.33 \mathrm{MPa})$ fueron las que obtuvieron un valor semejante a las elaboradas con poliestireno espumado (6.88MPa), por lo que ambas, presentaron casi la misma tendencia de regresar a su forma original al ser sometidas al ensayo de elasticidad.

Para la propiedad de tenacidad, los acondicionadores disminuyen su valor, ya que la tenacidad de las esponjas sin acondicionar fue la misma que la tenacidad de las probetas elaboradas con poliestireno espumado, por lo que, todas las probetas acondicionadas, 
presentaron una menor capacidad de absorber energía antes de romperse que las que no fueron acondicionadas.

Para la prueba de tensión en la carga máxima, no se encontraron valores reportados en la literatura para el poliestireno espumado de uso comercial, por lo que, sólo pudo realizarse la comparación a nivel estadístico.

\section{Conclusiones}

Fue posible obtener esponjas de quitina a partir de cefalotórax y exoesqueletos de camarón y, una vez acondicionadas las probetas de quitina, fue posible realizar su caracterización física a través de pruebas mecánicas (tensión en la carga máxima, tensión en el punto de ruptura, Módulo de Young, porcentaje de deformación, tenacidad y límite elástico), determinándose que el agente aditivo que más semejanzas le confirió a las probetas de quitina con el poliestireno espumado, fue el sorbitol. La característica que posee este aditivo frente a los otros dos probados, es el mayor número de grupos oxidrilo $(\mathrm{OH}-)$ en su molécula, lo que le permite interactuar mejor con los grupos funcionales de la espuma de quitina.
Continuará la investigación para buscar nuevos y mejores plastificantes que permitan obtener esponjas de quitina que puedan sustituir al poliestireno espumado en los usos generales que tiene este polímero poco biodegradable.

\section{Reconocimientos}

Los autores agradecen al personal del Instituto de Investigaciones en Materiales de la UNAM, por su valioso apoyo técnico y asesoría para realizar las pruebas mecánicas de los materiales.

Asimismo, por el apoyo financiero para adquirir reactivos y consumibles, agradecen al proyecto del Programa denominado PAPIME Clave PE101709 "Apoyo a la enseñanza experimental de las asignaturas terminales de las carreras que se imparten en la Facultad de Química de la UNAM" de la DGAPA-UNAM, con el que los tres primeros autores cursaron su asignatura terminal "Laboratorio de Desarrollo Experimental de Alimentos" (LABDEA), actualmente "Estancias Estudiantiles" (EE), antecedente de sus tesis profesionales. Se agradece el apoyo financiero parcial del Programa de Apoyo a la Investigación y el Posgrado, PAIP, de la Facultad de Química de la UNAM.

\section{Referencias bibliográficas}

1. CONAPESCA. (2005). Anuario Estadístico de Acuacultura y Pesca. SAGARPA. México D. F. México. 2005.

2. Cañipa, A., Durán, M.C., Escobedo, G., García, R. (1994). Aprovechamiento integral de cefalotórax de camarón. Serie "Tecnologías más limpias". Vol. 3, UNAM, PIQAyQA. ISBN 968-36-4104-0. México, D.F.

3. Flores, R. (2008). Obtención y caracterización de esponja de quitina a partir de cefalotórax de camarón. Tesis de Doctorado, UNAM, México D.F.

4. Barrera-Rodríguez, S., Flores, R., Durán-Domínguez-de-Bazúa, M.C (2007). Extracción de quitina del cefalotórax de camarón para la elaboración de películas / Chitin extraction from shrimp cephalothorax and exoskeleton for films preparation. Volumen 10. Serie: Tecno-

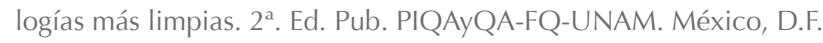

5. Arias, O. y Mendoza, A.M. (2010). Caracterización mecánica de un polímero de origen natural derivado de cefalotórax y exoesqueletos de camarón. Tesis de Licenciatura, Facultad de Química, UNAM, México, D.F. México. 
6. Bárcenas, E.M. (2010). Biopolímeros de cefalotórax y exoesqueleto de camarón. Uso de aditivos químicos para modificar sus propiedades mecánicas. Tesis de Licenciatura, Facultad de Química, UNAM, México, D.F. México.

7. Flores, R. (2004). Bioplástico de quitina. Formación de películas de quitina a partir de desechos de camarón por métodos ecológicos. Tesis de Maestría, UNAM, México, D.F.

8. Flores, R., Barrera-Rodríguez, S., Durán-Domínguez-de-Bazúa, M.C. (2004). Extracción ecológica de quitina y subproductos. Solicitud de registro de patente: Octubre 1. Facultad de Química, UNAM. Instituto Mexicano de la Protección Industrial. IMPI. Patente Número 264482. Otorgada el 12 de febrero de 2009. México, D.F. México.

9. ASTM. (2002). Standard Test Method for Tensile Poperties of Thin Plastic Sheeting D882-02. American Standard Testing and Materials. ASTM International, West Conshohocken, PA, EEUU. 